\title{
¿ES LA CONCIENCIA FENOMÉNICA UNA CONDICIÓN NECESARIA PARA LA INTENCIONALIDAD? LIMITACIONES DEL INSEPARATISMO FENOMENALISTA
}

\author{
Asier Arias Domínguez \\ IES Rosa Chacel, Madrid \\ http://dx.doi.org/10.15304/ag.38.1.4408
}

\section{Resumen}

Una de las principales líneas divisorias dentro del debate en torno al problema de la conciencia deja a un lado la postura del separatismo representacionalista y al otro la del inseparatismo fenomenalista. Según la primera, pueden existir estados mentales representacionales en ausencia de conciencia fenoménica y, además, una adecuada teoría naturalista de la representación es necesaria y suficiente para la explicación de la conciencia fenoménica. De acuerdo con la segunda, la conciencia fenoménica es necesaria para la existencia y la explicación de cualquier estado representacional y, de hecho, de cualquier estado mental. Varios argumentos han tratado de ofrecer respaldo a esta tesis inseparatista. Los propuestos por Galen Strawson y John Searle ilustran la estrategia compartida por dichos argumentos, basados en la introducción de premisas implícitas. A este problema se suman la inoperancia e inadecuación empírica del inseparatismo y asimismo su falta de verosimilitud filogenética. Palabras clave: inseparatismo, representación, mente, conciencia, intencionalidad.

\begin{abstract}
One of the main dividing lines within the debate on the problem of consciousness comes between representationalist separatism and phenomenalist inseparatism. According to the former, representational mental states are possible in the absence of phenomenal consciousness, and furthermore, an adequate naturalistic theory of representation is necessary and sufficient for the explanation of phenomenal consciousness. According to the later, phenomenal consciousness is necessary for the existence and the explanation of any representational
\end{abstract}

Recibido: 11/05/2017. Aceptado: 20/12/2017. 
state and, indeed, of any mental state. Several arguments have tried to offer support to this inseparatist thesis. Those proposed by Galen Strawson and John Searle summarize this kind of argument, which is based on the introduction of implicit premises. In addition to this failure, inseparatism faces the problems of its empirical inoperativeness and inadequacy, as well as its lack of phylogenetic verisimilitude.

Keywords: inseparatism, representation, mind, consciousness, intentionality.

\section{Introducción: inseparatistas frente a representacionalistas}

Los fenómenos mentales, ha venido asumiéndose en filosofía de la mente, se caracterizan de forma exclusiva y exhaustiva por su capacidad representacional y su aspecto cualitativo o fenoménico. A este supuesto se suma habitualmente el de que, de algún modo, una irreprochable explicación científica de la capacidad representacional de la mente podría algún día elaborarse dentro del marco de alguno de los diversos paradigmas que conviven en ciencias cognitivas, mientras que tal cosa resulta mucho más remota e improbable para el caso de su aspecto fenoménico. En línea con este supuesto, autores como Daniel Dennett, Fred Dretske, Gilbert Harman, William Lycan o Michael Tye han venido argumentando que la mente fenoménica puede reducirse a la representacional, la cual, a su vez, podría naturalizarse de un modo u otro, mostrándose dócil a su incardinación en el marco de una teoría científicamente respetable. Frente a esta corriente representacionalista, autores como Ned Block, David Chalmers, Brian Loar, Christopher Peacocke o John Searle han venido defendiendo la autonomía e irreductibilidad del aspecto fenoménico de los estados mentales.

¿Ha de ser una teoría naturalista de la representación la base para una explicación satisfactoria de lo mental en general y de su aspecto fenoménico en particular? ¿O hemos de entender, por el contrario, dicho aspecto fenoménico como un rasgo esencial de todo fenómeno mental en general y representacional en particular? El reduccionismo representacionalista gozó de una amplia aceptación entre los filósofos analíticos de la mente de las dos últimas décadas del siglo XX, que trataron de elaborar la teoría naturalista de la representación a la que refiere la primera pregunta y derivaron de ella esquemas conceptuales abstractos -y ajenos a la necesidad empírica de operacionalización - para dar cuenta del modo en que la misma serviría de base para la explicación del aspecto fenoménico de los estados mentales. Por su parte, los partidarios del inseparatismo responden afirmativamente no a la primera, sino a la segunda pregunta, introduciendo en la filosofía analítica de la mente una perspectiva que predominara tanto en la filosofía moderna, de Descartes a Locke, como en la tradición fenomenológica y 
según la cual la idea misma de fenómenos mentales independientes de la conciencia resulta contradictoria. ${ }^{1}$

Ha venido considerándose, pues, que dado que lo fenoménico es una esfera de lo mental que no sabemos muy bien de qué modo cabría integrar en un marco explicativo naturalista, sólo nos caben dos opciones: tratar de probar a) que puede reducirse a otra hipotéticamente concreta, discreta y aproblemática (la representacional), o b) que, sencillamente, es autónoma e irreductible y, de hecho, más básica que la representacional.

La primera opción se enfrenta a una serie de problemas que no abordaremos en este artículo (cf. Arias Domínguez, 2016, cap. 8). Apuntemos sólo de pasada al principal entre los mismos. No existiendo consenso acerca de los supuestos en que debiera basarse una teoría naturalista de la representación, la de comenzar a construir la casa por el tejado elaborando especulativos entramados conceptuales dentro de los cuales se presente lo fenoménico como en principio explicable en términos representacionales constituye una opción obviamente abierta a la discusión acerca de sus fundamentos.

Así las cosas, la segunda opción podría presentársenos como más atractiva, pero lo cierto es que, como comprobaremos, tampoco se trata de una alternativa exenta de problemas fundamentales. Los autores que rechazan

${ }^{1}$ El debate en torno a la naturalización de lo mental es de hecho más amplio y complejo y no se restringe exclusivamente a la disyuntiva planteada en los anteriores interrogantes. Así, por ejemplo, la tradición representacionalista a la que se enfrenta el inseparatismo ha sido recientemente desafiada desde posturas dinamicistas (cf. Chemero, 2009), encarnadas (cf. Gallagher, 2005) y enactivas (cf. Varela, Thompson, Rosch, 1991). Tomemos el caso del enactivismo, base del enfoque neurofenomenológico en el estudio de la conciencia (Varela, 1996). En el seno de esta tradición, la relación entre lo fenoménico y lo intencional, articulada en torno a la noción de autoconciencia pre-reflexiva, adquiere matices diversos de los habituales en la literatura sobre filosofía de la mente y ciencias cognitivas de raigambre analítica. Sin ir más lejos, lo habitual en la tradición analítica es equiparar intencionalidad y direccionalidad o referencia a un objeto, una equiparación en virtud de la cual autores como Searle o Strawson niegan que experiencias conscientes tales como las sensaciones corporales sean intencionales, mientras para el neurofenomenólogo sí lo serían "en el sentido fenomenológico más amplio de estar abiertos a lo otro o tener un carácter fenoménico que involucra al mundo" (Thompson, Lutz \& Cosmelli, 2005, p. 50). Con todo, y a pesar de importantes matices, la controversia acerca de la relación entre mente fenoménica y mente representacional tal y como se presenta en el debate entre representacionalistas e inseparatistas ha continuado su curso, como era de esperar, en buena medida al margen de la irrupción de estas propuestas antirrepresentacionalistas. La inclusión de los matices que sobre el debate del que nos ocuparemos arrojan dichas propuestas excede las posibilidades de este trabajo, que dedicamos a la discusión de las limitaciones del inseparatismo perfilando sus contornos mediante su contraste con la tesis a la que se contrapone, a saber, el reduccionismo representacionalista. 
la naturalización del aspecto fenoménico de lo mental a través de su reducción al representacional, o bien sostienen que lo fenoménico no puede, por principio, explicarse científicamente y abrazan el misterianismo, o bien que no puede reducirse a lo representacional porque, de hecho, constituye un prerrequisito para su existencia. Ésta es, justamente, la tesis inseparatista cardinal: la presencia de conciencia fenoménica es una condición necesaria para la existencia de estados intencionales o representacionales. ${ }^{2}$ Dedicamos los apartados que subsiguen al análisis y crítica de los principales intentos de elaboración de esta tesis: los pergeñados por Galen Strawson y John Searle.

La voz «inseparatismo» ha sido recientemente acuñada (cf. Horgan \& Tienson, 2002) para hacer referencia a este tipo de planteamiento en el que la intencionalidad no aparece como una propiedad básica o autónoma. ${ }^{3}$ El inseparatismo es, hasta cierto punto, una perspectiva heterodoxa, por cuanto en las últimas décadas la práctica habitual en filosofía de la mente, y en las ciencias cognitivas en general, ha consistido en tratar a la intencionalidad como ontológica y metodológicamente independiente y diferenciable de la conciencia fenoménica, asumiendo que son posibles los estados intencionales no fenoménicamente conscientes. Con todo, al margen de la apertura de nuevos horizontes que toda heterodoxia trae consigo, con ella no es suficiente. Comprobaremos a continuación que esta heterodoxia se muestra, como sugeríamos, incapaz de ofrecer respuestas satisfactorias a cuestiones cruciales dentro del debate contemporáneo en torno al problema de la conciencia.

\section{El inseparatismo de Strawson}

Strawson entiende que no todo estado mental dotado de aspecto fenoménico debe ser catalogado como intencional (Strawson, 1994/2010, p. 177). Así, apunta, muchos estados mentales no parecen tener un objeto definido, representarlo o dirigirse a él (sensaciones corporales y estados

${ }^{2}$ Usamos en lo sucesivo, y de acuerdo con la norma, ambas nociones como sinónimas. En lengua inglesa es habitual asimismo el uso indistinto de «intentionality» y «aboutness», refrendado en Dennett \& Haugeland (1987) y asumido por inseparatistas como Strawson (1994/2010).

${ }^{3}$ Mientras autores como Horgan y Tienson usan la noción de inseparatismo en sentido fuerte, significando que lo intencional implica a lo fenoménico y viceversa, aquí la usaremos en sentido débil, haciendo referencia a la idea de que la conciencia fenoménica es una condición necesaria para la existencia de estados intencionales. 
anímicos son los ejemplos habituales en este punto) y nadie dudaría de su carácter mental. Independientemente del crédito que uno esté dispuesto a conceder a esta idea en tanto que planteamiento fenomenológico descriptivo -inseparatistas como Horgan \& Tienson (2002) la rechazarían, al igual que lo harían representacionalistas como Tye (1995) o Bain (2003)-, Strawson extrae de la misma la conclusión de que la intencionalidad no ha de ser concebida como el objeto prioritario y fundamental del estudio de lo mental, dado que resulta desorientador presumir que solucionar el supuesto problema que comporta suministrará la clave que permita partir hacia una adecuada comprensión de la mente.

El punto de partida del inseparatismo de Strawson es su tesis de la ausencia de problema. Según la misma, de cara a ofrecer una explicación naturalista de la mente, la intencionalidad no se muestra problemática, esto es, no hay en ella problema alguno distinto del problema de la conciencia fenoménica. En otras palabras, la intuición que Strawson se propone articular es la de que ofrecer una explicación de la intencionalidad no supone una amenaza para una concepción fisicalista de le mente, al menos no una distinta de la que supone la conciencia fenoménica -0 , en sus términos, la experiencia consciente- ${ }^{4}$ La tesis de la ausencia de problema consta así de dos partes. La primera sostendría que la intencionalidad no supone un problema para una explicación naturalista de la mente. La segunda, por su parte, vendría a plantear que la experiencia consciente desafía el marco naturalista o se muestra dentro del mismo problemática, y como ella se encuentra esencialmente vinculada con la intencionalidad, entonces ésta, ahora sí, supone un desafío para el naturalismo, pero no por sí misma, sino por cuanto ella es, de algún modo, dependiente de la experiencia consciente.

Strawson (1994/2010, cap. 7) desarrolla su tesis de la ausencia de problema a través de una prolija serie de análisis conceptuales y experimentos mentales cuyo vínculo con la misma resulta a menudo difuso. El soporte argumental que la referida serie ofrece a la tesis de la ausencia de problema tiene la forma de una iteración: Strawson introduce su intuición de que el aspecto fenoménico de lo mental es necesario para la intencionalidad en el

${ }^{4}$ Hablamos de "intuición" dado que, ya en la primera edición de Mental Reality, Strawson plantea esta idea en esos términos, y lo hace en un tono que se verá matizado, por ejemplo, en el apéndice a la segunda edición -y no sólo en él, sino también en la propia primera edición, conforme avanza su argumentación en el capítulo séptimo-. Así pues, nos encontramos ante una declaración de intenciones muy poco ambiciosa: Strawson nos dice que se limitará a examinar una intuición acerca de cuya pertinencia, asegura, no pretende convencer a nadie (Strawson 1994/2010, p. 178). 
contexto de diferentes ejemplos esencialmente equivalentes, cuya forma y objetivo compartidos quedan solventemente compendiados en el siguiente experimento mental. Strawson nos propone que consideremos un objeto al que no atribuiríamos estados fenoménicamente conscientes: un misil termodirigido que sigue su blanco. ¿Posee intencionalidad semejante dispositivo? Algunos, como Searle, dirían cosas tales como que su intencionalidad es derivada de la de sus diseñadores (cf. Searle, 1992; 2004), pero Strawson plantea la cuestión desde el punto de vista de los "artefactos no-artificiales": consideremos que el misil surge espontáneamente programado por un extraño azar cósmico y hagámonos la misma pregunta. Si atribuimos referencialidad a la representación de su objetivo que tendría este "artefacto no-artificial", entonces separamos tajantemente la cuestión de la intencionalidad de la de la experiencia consciente, por cuanto le adscribimos aquélla aun cuando le negamos ésta, con lo cual ofrecemos respaldo a la tesis de la ausencia de problema. Pero Strawson se deshace enseguida de dicho respaldo al sugerir que si estamos dispuestos a atribuir intencionalidad a semejante dispositivo acabaremos atribuyendo intencionalidad a cualquier cosa, y al deshacerse de este modo del referido respaldo pone de manifiesto que la tesis que verdaderamente le interesa defender no es la de la ausencia de problema, sino la de la necesidad de la experiencia consciente para la intencionalidad.

Strawson utiliza estos experimentos mentales basados en "artefactos noartificiales" para hacer plausible su intuición de que no podríamos atribuir intencionalidad a tales objetos más que metafóricamente, pues sólo reaccionan de determinadas maneras que podemos interpretar como signos de intencionalidad, una intuición que descansa sobre la idea de que la diferencia entre la irreferencialidad de tales objetos y la referencialidad de nuestros estados mentales estriba sólo en la experiencia consciente, la cual, propone Strawson, sin duda marca la diferencia (Strawson, 1994/2010, p. 191). Cuando Strawson afirma que sus "artefactos no-artificiales" "sólo reaccionan de determinadas maneras" está hablando de lo que denomina "intencionalidad conductual", de la cual nos dice es que no es verdadera intencionalidad, pues, independientemente de la refinación de la conducta que sea el caso y de nuestra propia definición de la noción de conducta, faltará siempre el ingrediente esencial: la experiencia, sin la cual cualquier sistema podrá, meramente, comportarse como si tuviera intencionalidad: "Como muchos otros, creo que la mera intencionalidad conductual nunca puede equivaler a verdadera intencionalidad, por compleja que sea la conducta, y que no puede tenerse intencionalidad a menos que se sea un ser experiencial” (Ibíd., p. 208). 
A lo ya apuntado añade Strawson (Ibíd., pp. 203 y ss.) 1) que muchos se sienten inclinados a decir que mientras que sus pensamientos se refieren verdaderamente a algo, los de una "máquina" (un «ser no experiencial», en los términos que utiliza Strawson) no, 2) que muchos se sienten inclinados a decir que sólo en el caso experiencial se da verdadera intencionalidad, 3) que ésta es una intuición de gran alcance, y 4) que lo único que la experiencia consciente añadiría al caso de los seres no experienciales sería la propia experiencia. De este modo, concluye, la intencionalidad consiste en experiencia consciente sumada a todas las propiedades, capacidades y disposiciones no experienciales que nosotros (locus de la verdadera intencionalidad) compartimos con las máquinas o seres no experienciales. En este sentido, Strawson ha llegado a aseverar que "las únicas entidades verdaderamente intencionales son los episodios experiencialmente conscientes" (Strawson, 2005, p. 41), de donde concluye que la intencionalidad es un fenómeno esencialmente experiencial y que no pueden hallarse verdaderos fenómenos intencionales donde no hay experiencia consciente (Ibíd., p. 60). Así, la ocurrencia de un estado mental y, a fortiori, la ocurrencia de un estado intencional puede acaecer, exclusivamente, en un sujeto capaz de experimentar estados fenoménicamente conscientes, cosa que, según Strawson, no requiere de ulteriores justificaciones dado que, entiende, se trata de un hecho obvio acerca de lo que la palabra «mental» significa (Ibíd., p. 46).

La argumentación de Strawson en favor de la tesis inseparatista cardinal consiste en una serie de ejemplos destinados a mostrar intuitivamente que la experiencia consciente es una condición necesaria para la intencionalidad. No obstante, sus análisis conceptuales y experimentos mentales dan por supuesto, antes que demostrar, que la diferencia entre la irreferencialidad de sus "artefactos no-artificiales" y nuestra referencialidad estriba sólo en la experiencia consciente, la cual, sostiene, sin $d u d a$ marca la diferencia, con lo cual resulta complicado distinguir en su argumentación las premisas de la conclusión, que aparece como un supuesto implícito en aquéllas.

\section{El inseparatismo de Searle}

Searle defiende su postura inseparatista mediante su "principio de conexión", que postula: "la adscripción de un fenómeno intencional inconsciente a un sistema implica que el fenómeno es en principio accesible a la conciencia" (Searle, 1990, p. 586). En otras palabras, según el principio de conexión "todo estado intencional inconsciente es potencialmente consciente" (Shani, 2007, p. 66). El principio de conexión, tal y como Juan Her- 
moso y Pedro Chacón han señalado, "ocupa un lugar central en la teoría de la mente de Searle" (Hermoso \& Chacón, 2000, p. 172). No en vano, dicho principio constituyó el eje en torno al cual girara la reformulación de Searle de su hipótesis del trasfondo (cf. Searle, 1992), una reformulación muy significativa, dado que con ella la concepción searleana del inconsciente encuentra una formulación definitiva en la cual la noción de mente o intencionalidad inconsciente $-\mathrm{y}$ con ella toda noción de lo mental-aparece supeditada a la de mente consciente.

El principio de conexión fue presentado por vez primera en Searle (1989), y después en Searle (1990) y Searle (1992). Searle prepara en esta última versión el terreno para la aquiescencia mediante una serie de acotaciones que hacen las veces de exordio a su argumento, pues el principio de conexión tiene la forma de un argumento aunque, pide Searle, no debe ser comprendido como una deducción a partir de axiomas. En el contexto de dichas acotaciones, Searle acusa, sin citar ni comentar explícitamente ninguna propuesta concreta, a todo intento de naturalización de la intencionalidad de formar parte de una taimada ortodoxia cuya finalidad es la de construir una ciencia objetiva de la mente a la que le sea dable rehusar enteramente toda apelación a la experiencia consciente, y frente a tal ortodoxia Searle se presenta a sí mismo como un disidente solitario. El siempre elocuente Daniel Dennett describe esta autopercepción de Searle en los siguientes términos: "[Searle] se ve a sí mismo como un iconoclasta, librando una batalla solitaria contra «la tradición»" (Dennett, 1993, p. 193).

Searle constata, pues, que en las últimas décadas se ha realizado un esfuerzo considerable por llevar a cabo un proyecto que considera desorientado y confundente: el de separar intencionalidad y conciencia fenoménica e intentar dar cuerpo a una teoría de la intencionalidad en la cual la experiencia consciente no juegue ningún papel. "La idea es tratar la intencionalidad 'objetivamente', tratarla como si los rasgos subjetivos de la conciencia no importaran realmente" (Searle, 1992, p. 153). Un tratamiento análogo de la intencionalidad da cabida a estados y procesos mentales que de ningún modo se asemejan a los estados y procesos mentales que efectivamente experimentamos, y a Searle eso no acaba de parecerle del todo razonable, porque, desde su perspectiva, y dramatizando las consecuencias últimas de la misma, tener mente, ser sujeto pasible de adscripción de predicados mentales, es tener esto que los seres humanos experimentamos. De este modo, Searle desacredita la posibilidad de intencionalidades y mentalidades subpersonales rebatiendo entre líneas (ni cita, ni nombra, ni argumenta explícitamente al respecto) propuestas del tipo de la inferencia incons- 
ciente en psicología de la percepción (que se remonta a mediados del siglo XIX, en concreto, al Handbuch der Physiologischen Optik que Herman von Helmholtz publicara entre 1856 y 1867) o del tipo de las reglas sintácticas innatas de la psicolingüística de inspiración chomskiana. Searle no cita ningún trabajo concreto, pero alude a esta clase de planteamientos en psicología de la percepción y en psicolingüística por lo que ellos comparten, a saber, el recurso a estados y procesos representacionales que pretenden ser mentales pero que nada tienen que ver con lo que experimentamos como mental sino que tienen, de hecho, vetado el acceso a la conciencia. En otras palabras, Searle rechaza estas propuestas por su suscripción de una tesis según la cual la intencionalidad es ontológica y metodológicamente independiente de la experiencia consciente. Asimismo, en Searle (1990, p. 589 ) encontramos que, nuevamente sin hacer referencia o rebatir de forma explícita ninguna propuesta teórica concreta, Searle desacredita la posibilidad de la existencia de modelos mentales -en alusión a Jonhson-Laird (1983)-, de imágenes 2D -en alusión al pionero modelo computacional de la visión de Marr (1982)- o de un lenguaje del pensamiento -en alusión a Fodor (1975).

Una vez delimitado el objetivo de sus críticas, Searle pregunta: ¿qué es lo que hace que algo sea mental aun cuando no es consciente? Por toda respuesta: que de algún modo podría ser consciente. Esta respuesta es el principio de conexión. Antes de entrar en materia, Searle dice que tenemos profundas razones para creer que, efectivamente, las cosas son como esta respuesta las pinta. Una de esas profundas razones que Searle alega como pretexto para la formulación de su principio de conexión es que no podemos habérnoslas sin el poder explicativo del inconsciente (Searle, 1992, p. 151). No podemos prescindir de él, pero podemos -y es ésta, precisamente, la intención de Searle- plantear la noción del inconsciente como parasitaria de la noción de conciencia. En este sentido, Searle "intentará replantear la relación entre la conciencia y el inconsciente para mostrar (...) la centralidad de la conciencia con relación a lo inconsciente y la dependencia de la noción de inconsciente de la noción de conciencia" (García Valero, 2003, p. 67). Searle considera además perentorio que en el análisis de la intersección entre intencionalidad e inconsciente nuestra concepción del inconsciente nos permita 1) respetar la distinción entre estados genuina o intrínsecamente intencionales y estados que no lo son sino derivadamente, y 2) conservar también para los estados mentales inconscientes la aspectualidad inherente a toda intencionalidad, esto es, el hecho de que cada estado intencional representa sus condiciones de satisfacción (cf. Searle, 1983) solamente bajo 
ciertos aspectos. ${ }^{5}$ Searle resume este segundo punto diciendo que todo estado intencional tiene un cierto contorno de aspecto, cosa que, congruentemente, deberán tener asimismo los estados mentales inconscientes. Son estos dos extremos los que, según Searle, proporcionan la base para el argumento que ha de sostener la idea que denomina "principio de conexión".

Nos atendremos en nuestra exposición del referido argumento a la versión comprimida -en siete puntos, frente a los diez de la versión de 1989- y definitiva contenida en Searle (1992). Al igual que en la versión de 1990, y tal y como ya entonces señalara, el argumento es idéntico y, a pesar de los cambios, "su estructura básica se ha mantenido intacta" (Searle, 1990, p. 596) desde la primera versión hasta la definitiva. Recogemos y comentamos a continuación los señalados siete puntos (Searle, 1992, pp. 156-160).

1. Hay una distinción entre intencionalidad intrinseca e intencionalidad como-si; sólo la intencionalidad intrínseca es genuinamente mental.

2. Los estados intencionales inconscientes son intrínsecos.

3. Los estados intencionales intrinsecos, ya sean conscientes o inconscientes, tienen siempre contornos de aspecto.

4. El rasgo del aspecto no puede caracterizarse sólo, de manera exhaustiva o completa, en términos de predicados de tercera persona, conductistas, o incluso neurofisiológicos.

Searle afirma que la evidencia de tercera persona deja indeterminado el carácter de aspecto de los estados intencionales y asegura que habrá siempre entre la ontología del aspecto y los fundamentos epistémicos objetivos un vacío inferencial. Según Searle, pues, el contorno de aspecto de un estado intencional no puede ser descrito adecuadamente en términos de predicados de tercera persona, conductuales o neurofisiológicos (Searle 1990, p. 587). De ahí que, desde su punto de vista, ninguna acumulación o articulación de datos neurofisiológicos, objetivos, podrá ofrecer "datos de aspecto". Así, si Searle está en lo cierto, tan siquiera una neurobiología hipotéticamente completa y correcta permitiría determinar si una instancia concreta de pensamiento consciente sobre Héspero se refiere al lucero vespertino o al

\footnotetext{
${ }^{5}$ Según Searle, todo estado intencional está dotado de aspectualidad en tanto tiene un contenido y se refiere a él de un determinado modo: no se nos presenta nada íntegra y neutralmente, sino que cada vez que nos representamos algo lo hacemos en alguna modalidad (lo deseamos, lo recordamos, lo percibimos, lo creemos) y desde alguna perspectiva (cuando veo un objeto no lo veo desde todos los ángulos a la vez del mismo modo que cuando pienso en mi tía pienso en la mujer que dio a luz a mi prima, en la esposa de mi tío o en aquella agradable compañía aquel verano, pero no en todo esto a la vez).
} 
matutino, o si una instancia determinada de deseo de agua lo es de $\mathrm{H}_{2} \mathrm{O}$, cosa que requeriría de una inferencia de lo neurofisiológico a lo intencional que haría explícito el modo en que la especificación de lo neurofisiológico en términos neurofisiológicos no es aún una especificación de lo intencional.

5. Pero la ontología de los estados mentales inconscientes, en el momento en que son inconscientes, consiste enteramente en la existencia de fenómenos puramente neurofisiológicos.

En este punto surge una contradicción, pues si la ontología de la intencionalidad inconsciente consiste enteramente en hechos neurofisiológicos, objetivos, de tercera persona, $\mathrm{y}$, al tiempo, dicha intencionalidad posee el referido rasgo de aspecto, que no puede estar exclusivamente constituido por tales hechos, necesitamos -Searle necesita- añadir un ingrediente a esa ontología, y Searle, en un giro que trae a las mientes el escolástico -hemos de remontarlo en verdad al aristotélico De Anima (II, 1, 412 a 19)- "corporis physici organici potentia vitam habentis" (acendrado de perifollos: "cuerpo con vida en potencia”), repentiza el siguiente:

6. La noción de un estado intencional inconsciente es la noción de un estado que es un posible pensamiento o experiencia consciente.

Según Searle, para que los fenómenos mentales inconscientes sean genuinamente mentales, y, en sus términos, intrínsecamente intencionales, tienen que conservar de algún modo contornos de aspecto, y Searle encuentra que ello sólo puede lograrse postulando que esos fenómenos inconscientes son de hecho posibles estados fenoménicamente conscientes, que la intencionalidad de los mismos depende, pues, de su potencial fenomenalidad. Searle acusa que la noción problemática es en estas frases la de posibilidad (él la encuentra problemática porque un estado intencional del tipo de los que anda buscando, entiende, podría tener, después de todo, impedido su acceso a la conciencia), ${ }^{6}$ pero todo lo que hace respecto de dicho carácter problemático es remitir al séptimo y último punto de su argumento y presentarlo

${ }^{6}$ Searle habla de las causas de un impedimento semejante en términos de lesiones cerebrales o represiones psicológicas, a pesar de lo indefinido de la primera idea -que lleva a pensar en un ingenuo modelo espacial de la conciencia como el que Searle critica; así, cuando impugna el modelo de los peces-estados-mentales que nadan en el inconsciente y emergen idénticos a la superficie parece tener en mente algo parecido a lo que sugiere esta idea de un estado mental que cuenta con todo lo que hay que tener para acceder al reino de la mentalidad consciente, excepto porque se topa con que han cerrado el camino en algún punto del trayecto neuronal hacia la superficie- y del cuño psicoanalítico de la segunda -no hay que perder de vista que Searle contrapone su concepción del inconsciente tanto a la propia de la ortodoxia cognitivista como a la propia de la tradición psicoanalítica. 
como una explicitación adicional de éste (el sexto) implicada, además, por su conjunción con el anterior.

7. La ontología del inconsciente consta de rasgos objetivos del cerebro capaces de causar pensamientos conscientes subjetivos.

$\mathrm{Al}$ describir algo como un estado intencional inconsciente, dice Searle, caracterizamos una ontología objetiva en virtud de su capacidad causal de producir conciencia fenoménica, que Searle concibe, recordemos, como una "ontología subjetiva": caracterizamos de este modo, pues, una ontología objetiva en virtud de su capacidad causal de producir una ontología subjetiva. Sin atender a lo problemático de esta suerte de transducción cartesiana, Searle se limita en este punto a tratar de resolver la dificultad que implicaría una virtual imposibilidad para acceder a la conciencia por parte de un estado potencialmente consciente presentando la causalidad aquí operante en términos disposicionales. Tales disposiciones seguirían existiendo a pesar de un bloqueo del tipo de los que Searle contempla (vid. supra: nota al pie $\mathrm{n}^{\circ} 6$ ) al igual que un volumen dado de cloruro de sodio seguiría siendo soluble aun cuando jamás se humedeciera. "El concepto de intencionalidad inconsciente es entonces el de latencia relativa a su manifestación en la conciencia" (Searle, 1992, p. 161). ${ }^{7}$

Searle resume su argumento y los motivos que le condujeran a la formulación del mismo como sigue. La noción de inconsciente (concebida por él como el conjunto completo de las actitudes proposicionales que un sujeto tiene mientras no es consciente de ellas) es valiosa desde el punto de vista explicativo. Hemos pues de preservarla. Pero mientras un estado mental permanece inconsciente no hay nada respecto del mismo aparte de neurofisiología. ¿Cómo entenderlo entonces como intencional -y si, como en su modelo, nos hallamos ante actitudes proposicionales han de serlo forzosamente- cuando el contorno de aspecto es por un lado inherente a todo estado intencional y por otro permanece ausente en el nivel neurofisiológico? La solución de Searle consiste en postular que el contorno de aspecto puede ser atribuido a las estructuras y procesos neurofisiológicos pertinentes sólo si entendemos que los mismos tienen la capacidad de producir estados fenoménicamente conscientes. La diferencia entre los estados neurofisiológicos que nada tienen de mentales y los estados neurofisiológicos inconscientes pero mentales estaría de este modo en que sólo estos últimos son candidatos para la conciencia. Lo mental se mostraría así como neurofisiología más conciencia fenoméni-

\footnotetext{
${ }^{7}$ Cursivas en el original.
} 
ca -bien se halle ésta en acto o en potencia-, a pesar del modo en que Searle trata de conciliar ambas nociones presentándolas como intercambiables en su intento de eludir el dualismo (cf. Searle, 2004, p. 124). La conclusión es, en cualquier caso, que los estados mentales por principio inaccesibles a la conciencia no existen, siendo así que, en la propuesta de Searle, "carece de sentido la postulación llevada a cabo por científicos cognitivos y filósofos de la mente de estados mentales que (...) no guardan relación con la experiencia subjetiva" (Hermoso \& Chacón, 2000, p. 175).

Examinaremos a continuación las dificultades a las que no logra hacer frente el inseparatismo searleano en su intento de presentar a la conciencia fenoménica como una condición necesaria para la presencia de intencionalidad. A tal fin, comencemos por esclarecer brevemente el enredo conceptual que subyace al argumento que trata de ofrecer sustento al principio de conexión. Searle pretende convencernos de que la intencionalidad depende de lo fenoménico, pero en los puntos 3 y 4 de su argumento se introduce la noción de contorno de aspecto como una especie de comodín. Como el contorno de aspecto es, según Searle, esencial para la intencionalidad, y como el mismo no puede, idénticamente, estar ausente en ningún estado fenoménicamente consciente, la conclusión de Searle está implícita en sus definiciones de las nociones que involucra en sus premisas -ha llegado en este punto a afirmarse que la definición habitual de conciencia fenoménica apenas dista de la que Searle ofrece de su noción de contorno de aspecto (Gunson, 1998, p. 156)-. "El argumento de Searle depende de la afirmación de que sin conciencia no hay manera de explicar el contorno de aspecto que exhibe la intencionalidad" (Chalmers, 1996, p. 334), y es precisamente esta asunción implícita la que el argumento de Searle debiera respaldar, en lugar de darla por supuesta.

En la argumentación de Searle sólo lo fenoménico podría proporcionar a la intencionalidad contorno de aspecto, pero, al constituir éste una característica definitoria tanto de la intencionalidad como de la conciencia fenoménica, el argumento podría funcionar, si lo hiciera, en ambas direcciones. En otras palabras, Searle no consigue elucidar en qué medida los fenómenos mentales son intrínsecamente intencionales a causa de lo fenoménico por cuanto hace depender idénticamente a lo intencional y lo fenoménico de su noción de "contorno de aspecto". De este modo, nada más allá de asunciones previas no tematizadas puede favorecer uno u otro orden explicativo o de dependencia.

El intento de Searle de probar que "sólo un ser capaz de conciencia puede tener estados intencionales" (Searle, 2006, p. 104) dado que la conciencia 
fenoménica es un prerrequisito de la intencionalidad consiste, al igual que el resto de los conatos emprendidos en esta dirección, en una explicitación de mayor o menor profundidad de intuiciones y asunciones previas no tematizadas y, nuevamente al igual que el resto de propuestas similares, en ningún caso en una inconcusa demostración o una guía eficaz para la obtención o interpretación evidencia empírica.

En este sentido, esto es, en lo tocante a la interpretación de la evidencia empírica, cabe notar que la visión ciega (cf., v. g., Weiskrantz, 2007) o el más simple paradigma experimental de priming (cf., v. g., Neumann \& Klotz, 1994) ofrecen datos difícilmente articulables dentro del marco searleano, pues hablar de los fenómenos mentales inconscientes que afectan a la conducta de los sujetos en cualquiera de ambas condiciones como intencionales dada su potencialidad consciente supondría que tanto la lesión en $\mathrm{V} 1$ como el escotoma en el campo visual de un paciente con visión ciega debieran poder desaparecer, como asimismo que los sujetos experimentales de estudios de priming debieran poder diferenciar los estímulos que producen los contrastes entre sus respuestas ante las diversas situaciones experimentales. Sobra añadir que, en ambos casos, se trata de posibilidades imposibles, si se nos permite el oxímoron. Hablar de potencialidad sólo tiene sentido cuando la misma puede de hecho actualizarse, cosa que no sucede con la presunta potencialidad consciente de la actividad nerviosa que de los geniculados laterales parte hacia zonas circunstriadas sin pasar por V1 cuando a un paciente con visión ciega se le presentan estímulos visuales en el área ciega de su campo visual. ${ }^{8}$ Así pues, resulta notorio que la de la potencialidad consciente de un estado mental es, por decir lo menos, una noción problemática y, de este modo, un extraño lugar al que acudir en busca de una demostración del pretendidamente necesario vínculo entre intencionalidad y fenomenalidad.

\footnotetext{
${ }^{8}$ Nótese el error que entraña replicar utilizando el argumento de que los estados mentales implicados son potencialmente conscientes aunque no puedan de hecho actualizarse dado que, en "condiciones normales", podrían hacerlo, del mismo modo que un volumen dado de cloruro de sodio seguiría siendo soluble aunque permaneciera por siempre aislado de la posibilidad de disolverse, pues, en "condiciones normales", esto es, no encontrándose aislado de tal posibilidad, podría en cualquier caso disolverse. El error consiste en que los estados mentales implicados en los fenómenos de priming o en la visión ciega están siempre, por principio y en sus "condiciones normales" aislados de la posibilidad de hacerse conscientes. La analogía no funciona, pues, dado que para que lo hiciera todo el cloruro de sodio del universo debiera estar siempre, por principio y en sus "condiciones normales" aislado de la posibilidad de disolverse, de tal modo que no sería soluble, pues en tales circunstancias no podríamos atribuirle esa potencialidad.
} 
Por otra parte, de acuerdo con la posición de Searle, nada fue mental hasta que surgieron en la filogénesis animales capaces de sostener estados mentales fenoménicamente conscientes, una hipótesis nada obvia y, a la luz de la lógica evolutiva, poco plausible. La postura de Searle contradice en este punto la evidencia en que se basan las teorías neurobiológicas de la conciencia que más atención han prestado a la filogénesis, y muy particularmente desatiende el carácter intencional de la noción de mapa neuronal, que en todas ellas aparece como prerrequisito de las formas más básicas y arcaicas de conciencia fenoménica, como la conciencia primaria de Edelman, el proto sí mismo de Damasio o la conciencia afectivo-anoética de Panksepp, por ejemplo. Finalmente, partir del señalado modelo del inconsciente entendido como colección de actitudes proposicionales latentes implica tácitamente un compromiso chauvinista con una tesis según la cual lo fenoménico es necesario para la intencionalidad y, así, para lo metal en general, por su implicación en un aspecto de lo mental consistente en la capacidad de mantener actitudes hacia proposiciones, algo exclusivamente al alcance de criaturas lingüísticas como nosotros.

A la vista de esta serie de dificultades, el argumento de Searle en defensa de la tesis según la cual todo fenómeno mental se encuentra esencialmente vinculado con la conciencia se nos presenta antes como un dispositivo retórico destinado a resaltar el atractivo intuitivo de asunciones no tematizadas -sino dadas por sentadas- que como un argumento a favor de las mismas clara y sólidamente fundamentado y desarrollado.

\section{Conclusiones}

Hemos comprobado que los argumentos de Strawson y Searle a favor de la tesis inseparatista necesitan recurrir a la introducción de premisas implícitas. En el caso de Strawson, dicho recurso se materializa en una estrategia retórica que no permite distinguir entre premisas y conclusiones en el desarrollo de una serie de argumentos que habrían de probar que la diferencia entre irreferencialidad y referencialidad estriba sólo en la experiencia consciente, pero que remiten en el paso crucial de la demostración a la propia conclusión aduciendo como hecho incontrovertible que, efectivamente, la experiencia marca sin $d u d a$ esa diferencia. En lugar de buscar un respaldo empírico o argumental a sus intuiciones, Strawson las introduce subrepticiamente en sus premisas. La argumentación de Searle adolece de idénticas fallas, bien que en su caso se nos presentan en la forma de un embrollo 
conceptual ocasionado por el al menos parcial solapamiento de la extensión de sus nociones de conciencia fenoménica, contorno de aspecto e intencionalidad. En su caso, por otra parte, se hacen asimismo manifiestas la inconsistencia empírica del inseparatismo y su falta de verosimilitud filogenética.

A estas conclusiones podemos añadir, en una apretada síntesis en cinco puntos, los principales motivos -explícita o implícitamente contenidos en nuestra crítica de las posturas de Searle y Strawson- que debieran mantenernos alerta frente al encanto heterodoxo del inseparatismo.

1. Los partidarios del fenomenalismo inseparatista han venido mostrándose incapaces de sustentar sus tesis en evidencias empíricas, incapaces de abrir vías para la obtención o la interpretación de evidencia empírica y, lo que es peor, asimismo incapaces de articular cabalmente sus tesis con la evidencia empírica disponible.

2. Como hemos podido comprobar, las disquisiciones con las que han rebasado la mera iteración de la doctrina de la necesidad de lo fenoménico para la presencia de intencionalidad no consisten sino en ejemplos destinados a ilustrarla intuitivamente antes que a demostrar su adecuación o, en el mejor de los casos, en argumentos en cuyas premisas se introducen de contrabando las que habrían de ser sus conclusiones mediante premisas implícitas.

3. El inseparatismo, tal y como la propuesta de Searle pone elocuentemente de relieve, conduce a una dicotomía de todo o nada, a una concepción dualista de la realidad según la cual existen, por una parte, organismos conscientes y, por otra, ciegos dispositivos mecánicos en ningún sentido relacionados con nada que quepa denominar mental (cf. Shani, 2007). Existen en este sentido razones sólidas para poner en tela de juicio la idea de que la búsqueda de criterios estrictos de los que servirnos para delimitar de forma limpia y rígida los fenómenos mentales distinguiéndolos de sus precursores evolutivos vaya a ser una empresa exitosa, razones que tienen en último término que ver con el hecho de que resulta extremadamente difícil, sino imposible, encontrar en el árbol filogenético el lugar exacto en que la primera pluma se diferenció de la última escama o el momento exacto en que rompió el cascarón el primer ave o el último reptil.

4. El inseperatismo obliga a elegir entre emergencia súbita y panpsiquismo. En pocas palabras, si todas las características definitorias de lo mental han de venir juntas, sólo le quedan a lo mental dos opciones: o surgir abruptamente de lo físico entero y acabado, de repente y sin precedentes, 
o ser inherente a ello. Venimos así a parar en una suerte de catastrofismo cuvieriano como única alternativa a una concepción según la cual cada tipo de leptón experimenta su particular clase de deseos, creencias, ansiedades y picores.

El núcleo del inseparatismo consiste en definir la característica básica de cualquier estado intencional en términos de propiedades que no hay motivos ni teóricos ni empíricos que impidan considerarlas como filogenéticamente posteriores y, por tanto, explicativamente derivadas. Esto quiere decir que nada niega a priori la posibilidad de que dentro del reino animalia los estados internos de algún miembro del filo mollusca o la familia arthropoda fueran referenciales mucho antes de que ningún vertebrado alcanzara a ser fenoménicamente consciente.

El inseparatismo obliga pues, desde la perspectiva filogenética, a concebir la irrupción de experiencia consciente como condición necesaria para la adscripción de intencionalidad a cualesquiera estados de cualesquiera organismos. Esto, a su vez, fuerza a la elección entre 1) alguna suerte de panfenomenalismo según la cual incluso las formas más arcaicas de referencialidad presentes en el mundo biológico son verdaderamente intencionales en tanto en cuanto la experiencia consciente es un rasgo fundamental de la naturaleza que hallamos en todos y cada uno de sus órdenes y niveles, ${ }^{9} 2$ ) una estrecha definición ad hoc de intencionalidad (calificada entonces de «original» o «verdadera») según la cual la referencialidad que acaso da la sensación de que encontramos en organismos o procesos que tenderíamos a clasificar como inconscientes no tiene nada que ver con la "verdadera intencionalidad", exclusividad biológica de los organismos fenoménica-

\footnotetext{
${ }^{9}$ Esta opción trae consigo el nuevo problema de explicar los mecanismos por los cuales la conciencia ordinaria surge de las protoconciencias ex hypothesi diseminadas por todo lo ancho y largo del mundo natural (Lycan, 2006). Strawson ha venido en cualquier caso a desembocar en esta opción (cf. Strawson, 2006), que a su vez le ha conducido a la defensa de una extraña suerte de epifenomenalismo panpsiquista: "no hay una buena razón (...) para pensar que [la conciencia] surgió porque tuviera valor adaptativo. La selección natural necesita algo sobre lo que trabajar y sólo puede trabajar partiendo de aquello con lo que se encuentra. La experiencia/conciencia tenía que existir antes de que pudiera ser explotada y moldeada, tal como lo hizo la materia no experiencial. La tarea de dar una explicación evolutiva de la existencia de la conciencia es exactamente como la tarea de dar una explicación evolutiva de la existencia de la materia: no existe tal tarea. La selección natural moldea los fenómenos de la experiencia que encuentra en la naturaleza" (Strawson, 2010, p. 304). En textos posteriores, Strawson ha seguido presentando al panpsiquismo como la mejor opción tanto desde el punto de vista de la simplicidad teórica como desde el de la parsimonia ontológica (cf. Strawson, 2017).
} 
mente conscientes, o 3) una concepción de lo mental según la cual todas las propiedades mentales surgieron abrupta y simultáneamente, de forma que nada pudo contar como representación de nada hasta el momento en que surgieron organismos fenoménicamente conscientes, una doctrina nada obvia desde el punto de vista filogenético y, de hecho, enfrentada a las conclusiones acerca de la filogenia de lo mental sostenidas por las diferentes teorías neurobiológicas de la conciencia -de la síntesis del trabajo previo de Gerald Edelman que él mismo y Giulio Tononi llevaran a cabo a la teoría de la información integrada que Tononi desarrollara de forma independiente con posterioridad, de los planteamientos pioneros Francis Crick y Christof Koch a los que éste ha venido defendiendo recientemente en la línea de Tononi-, y muy particularmente por aquéllas que más atención han prestado, justamente, a la filogénesis desde la neurociencia cognitiva y la neurociencia afectiva (cf., v. g., Damasio, 2010).

5. Finalmente, cabe apuntar a la falta de fundamentación de las asunciones ontológicas y epistemológicas que tanto representacionalistas como inseparatistas vinculan con nuestra economía léxica (cf. Arias Domínguez, 2016, cap. 8). Esto es, bien cabe que «intencionalidad» y «fenomenalidad» no pasen de ser sustantivos utilizados para aludir a conjuntos un tanto heterogéneos de fenómenos cuya explicación dependa, así, de un plural conjunto de marcos teóricos. No obstante, la práctica habitual, común a representacionalistas e inseparatistas, consiste en tratar dichas nociones como conceptos netamente unívocos y perfectamente definidos que refieren a fenómenos completamente discretos y, así, explicables de acuerdo con un único marco teórico, conceptos en los que, además, por algún motivo no especificado, se asume que debiera basarse la explicación de todo fenómeno mental. Tampoco hemos podido hallar especificado en la bibliografía disponible el motivo por el cual hemos de suponer que la solución al problema de la conciencia depende de la especulación acerca de la relación entre los conceptos de intencionalidad y fenomenalidad. La idoneidad de cargar las tintas sobre estos supuestos esencialistas -inertes por lo que a la crítica y el desarrollo de marcos teóricos vinculados a programas experimentales respecta-, sobre los tradicionales enredos conceptuales vinculados a los mismos, y asimismo sobre los órdenes de dependencia -explicativos y constitutivos- asociados a dichos supuestos se restringe más si cabe ante la posibilidad abierta de orientar la labor investigadora en la filosofía de la mente implicada en los consciousness studies hacia el análisis conceptual en el contexto de la crítica informada de marcos teóricos concretos en su 
articulación con datos obtenidos en psicología, etología y neurociencias. Muchos nos inclinamos a pensar que la contribución de la filosofía de la mente a la solución del problema de la conciencia depende antes de esta clase de trabajo que de la acostumbrada especulación acerca de la relación entre dos abstracciones pretendidamente homogéneas y discretas, a saber, la intencionalidad y la fenomenalidad.

\section{Bibliografía}

Arias Domínguez, A. (2016) El problema de la conciencia en la filosofía de la mente y las ciencias cognitivas. Replanteamiento del núcleo del debate y valoración crítica de los principales marcos teóricos. Madrid: UCM.

Bain, D. T. (2003) "Intentionalism and pain", Philosophical Quarterly, vol. 53, no. 213, pp. 502-523. https://doi.org/10.1111/1467-9213.00328

Chalmers, D. J. (1996) The Conscious Mind: In Search of a Fundamental Theory. New York: Oxford University Press.

Chemero, A. (2009) Radical Embodied Cognitive Science. Cambridge, MA: MIT Press.

Damasio, A. R. (2010) Self Comes to Mind: Constructing the Conscious Brain. New York: Pantheon.

Dennett, D. C. (1993) "Review of Searle, The Rediscovery of the Mind", Journal of Philosophy, vol. 60, no. 4, pp. 193-205. https://doi. org/10.5840/jphil199390449 https://doi.org/10.2307/2940971

Dennett, D. C. \& Haugeland, J. (1987) “Intentionality”, en R. L. Gregory (ed.), The Oxford Companion to the Mind, Oxford, UK: Oxford University Press, pp. 383-386.

Fodor, J. A. (1975) The Language of Though. New York: Crowell.

Gallagher, S. (2005) How the Body Shapes the Mind. Oxford: Oxford University Press. https://doi.org/10.1093/0199271941.001.0001

García Valero, H. A. (2003) "La filosofía de la mente de John Searle”, Lógoi. Revista de Filosofía, no. 6, pp. 41-82.

Gunson, D. (1998) Michael Dummett and the Theory of Meaning. Avebury Series in Philosophy. Aldershot: Ashgate.

Hermoso, J. \& Chacón, P. (2000) "Sobre el carácter irreductible de la intencionalidad: la ontología del inconsciente y los dos conceptos de trasfondo en Searle", en P. Chacón Fuertes \& M. Rodríguez González (eds.), Pensando la mente. Perspectivas en filosofía y psicología, Madrid: Biblioteca Nueva, pp. 167-194. 
Horgan, T. \& Tienson, J. (2002) "The phenomenology of intentionality and the intentionality of phenomenology", en D. Chalmers (ed.), Philosophy of Mind: Classical and Contemporary Reading, Oxford, UK: Oxford University Press, pp. 520-533.

Johnson-Laird, P. N. (1983) Mental Models: Towards a Cognitive Science of Language, Inference, and Consciousness. Cambridge, UK: Cambridge University Press.

Lycan, W. (2006) "Resisting ?-ism”, Journal of Consciousness Studies, vol. 13, nos. 10-11, pp. 65-71.

Marr, D. (1982) Vision. A Computational Investigation into the Human Representation and Processing of Visual Information. San Francisco: W. H. Freeman \& Co.

Neumann, O. \& Klotz, W. (1994) "Motor responses to nonreportable, masked stimuli: Where is the limit of direct parameter specification?", en C. Umilta and M. Moscovitch (eds.), Attention and Performance XV. Conscious and Nonconscious Information Processing, Cambridge, MA: MIT Press. pp. 123-150.

Searle, J. R. (1983) Intentionality. An Essay in the Philosophy of Mind. Cambridge, UK: Cambridge University Press. https://doi.org/10.1017/ CBO9781139173452

Searle, J. R. (1989) “Consciousness, unconsciousness and intentionality”, Philosophical Topics, vol. 17, no. 1, pp. 193-209.

Searle, J. R. (1990) "Consciousness, explanatory inversion and cognitive science", Behavioral and Brain Sciences, vol. 13, no. 4, pp. 585-596. https://doi.org/10.1017/S0140525X00080304

Searle, J. R. (1992) The Rediscovery of the Mind. Cambridge, MA: MIT Press.

Searle, J. R. (2004) Mind: A Brief Introduction. New York: Oxford University Press.

Searle, J. R. (2006) "What is to be done", Topoi, vol. 25, no. 1, pp. 101-108. https://doi.org/10.1007/s11245-006-0016-z

Shani, I. (2007) "Consciousness and the first person. A critical appraisal of Searle's connection principle”, Journal of Consciousness Studies, vol. 14, no. 12, pp. 57-91.

Strawson, G. (1994/2010) Mental Reality (2nd ed.). Cambridge, MA: MIT Press, Bradford Books.

Strawson, G. (2005) "Intentionality and experience: Terminological preliminaries”, en D. W. Smith \& A. L. Thomasson (eds.), Phenomenology and Philosophy of Mind, Oxford, UK: Oxford University Press, 41-66. https://doi.org/10.1093/acprof:oso/9780199272457.003.0003 
Strawson, G. (2006) "Realistic monism: Why physicalism entails panpsychism”, Journal of Consciousness Studies, vol. 13, nos. 10-11, pp. 3-31.

Strawson, G. (2010) "Radical self-awareness", en M. Siderits, E. Thompson \& D. Zahavi (eds.), Self, No Self? Perspectives From Analytical, Phenomenological, and Indian Traditions, New York: Oxford University Press, pp. 274-307.

https://doi.org/10.1093/acprof:oso/9780199593804.003.0011

Strawson, G. (2017) "Physicalist Panpsychism", en S. Schneider \& M. Velmans (eds.), The Blackwell Companion to Consciousness, Chichester: John Wiley \& Sons, pp. 374-390. https://doi.org/10.1002/9781119132363. $\operatorname{ch} 27$

Thompson E., Lutz, A. \& Cosmelli, D. (2005) "Neurophenomenology: An introduction for neurophilosophers", en A. Brook \& K. Akins (eds.), Cognition and the Brain: The Philosophy and Neuroscience Movement, New York: Cambridge University Press, pp. 40-97.

Tye, M. (1995) Ten Problems of Consciousness. Cambridge, MA: MIT Press.

Varela, F. J., Thompson, E. \& Rosch, E. (1991) The Embodied Mind: Cognitive Science and Human Experience. Cambridge, MA: MIT Press.

Varela, F. J. (1996) "Neurophenomenology: A methodological remedy for the hard problem", Journal of Consciousness Studies, vol. 3, no. 4, pp. 330-349.

Weiskrantz, L. (2007) “The case of blindsight”, en M. Velmans \& S. Schneider (eds.), The Blackwell Companion to Consciousness, Oxford: Blackwell, pp. 175-180. 